\title{
Microwave photonics shines
}

\author{
The combination of microwave photonics and optics has advanced many applications in defence, wireless \\ communications, imaging and network infrastructure. Rachel Won talks to Jianping Yao from the University of \\ Ottawa in Canada about the importance of this growing field.
}

What is microwave photonics? Microwave photonics is a field that studies the interaction between microwave and optical waves for applications such as communications, radars, sensors and instrumentations. These applications require ever-increasing speed, bandwidth and dynamic range. They also need devices that are small, lightweight and low power, while exhibiting large tunability and strong immunity to electromagnetic interference. Digital electronics is the most widely used approach nowadays for these applications. Unfortunately, the speed of digital electronic is normally less than several gigahertz - a limit established primarily by the electronic sampling rate. The unique capabilities offered by photonics for processing ultrawidebandwidth, high-frequency microwave signals make it a promising alternative for wideband microwave signal processing.

\section{What applications drive the field?} Over the past 30 years, research activity in microwave photonics has focused on the generation, distribution, control and processing of microwave signals for defence applications. Some examples are low-phasenoise and high-frequency microwave sources, high-dynamic-range microwave photonic analog links, true-time-delay phased array antennas, frequency-tunable high- $Q$ microwave filters and high-speed analogto-digital convertors. The other driving force comes from broadband wireless access networks. Future networks will be expected to support wireless communications at data rates reaching multiple gigabits per second. Such speeds can be achieved by modulating digital data over a microwave carrier at the millimetre-wavelength band, such as the license-free $60 \mathrm{GHz}$ band. The extremely low loss and broadband width of state-ofthe-art optical fibres enable the distribution of $60 \mathrm{GHz}$ signals with high efficiency between pico- or femtocells. Furthermore, the extremely low power consumption of an access network comprised of pico- or femtocells would making it much greener than current macrocell networks, which require high-power base stations. In addition to 60 -GHz-over-fibre technology, broadband wireless access can also be achieved using

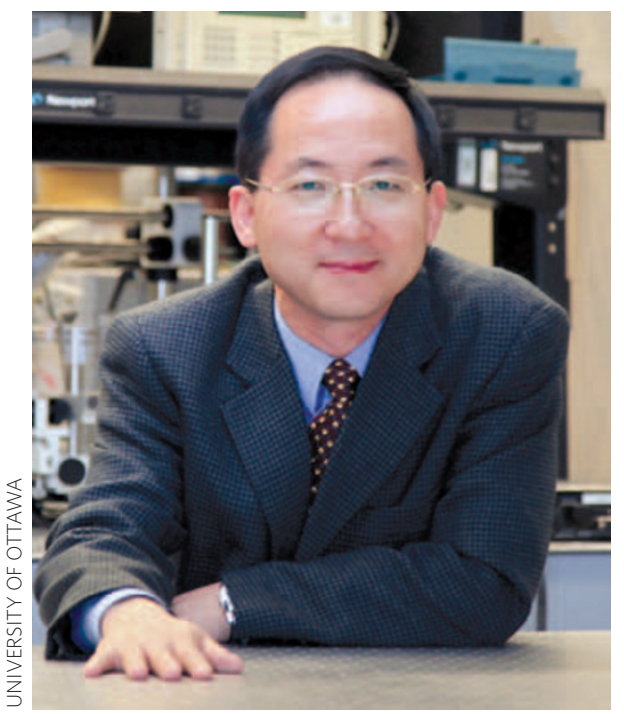

Yao: "The integration of microwave photonic components onto photonic-integrated circuits is crucial to the implementation of low-cost, advanced microwave photonics."

ultrawide-bandwidth-over-fibre technology. This is different from $60 \mathrm{GHz}$ technology in that it has the key advantages of being carrierfree and simple to implement, which greatly reduces installation costs.

What are the most recent applications? The first example is the use of microwave photonics for medical imaging. Microwave photonic solutions can be employed to generate high-quality and frequency-tunable terahertz waves by beating two optical wavelengths with a wavelength spacing that corresponds to a terahertz wave. Medical imaging with terahertz waves offers some unique advantages over other imaging modalities. First, it is not harmful to biological samples as the energy of a terahertz photon is one million times lower than that of an X-ray photon. Second, terahertz imaging provides spectroscopic information that is generally absent in optical, X-ray and nuclear magnet resonance images, as the unique rotational, vibrational and translational responses of molecules, radicals and ions is often in the terahertz frequency range. The second recent example of microwave photonics is for 'the internet of things', a relatively new term used to describe a global network infrastructure that links physical and virtual objects through the exploitation of data capture and communication capabilities. Using such a network, the information about an identifiable object can be collected, distributed wirelessly and then sent to the central office via optical fibres.

\section{How much of microwave photonics has} been commercialized?

Several companies have been established over the past few years to develop microwave photonic solutions for various applications. For example, Photonic Systems has developed microwave photonic links that operate at over $40 \mathrm{GHz}$ and offer a range of bandwidths, noise figures and dynamic ranges. Pharad has developed optimized-performance radiofrequency photonic transceivers for high-dynamicrange and low-loss radio-over-fibre transport. OEwaves, a spin-off from Caltech, has developed ultralow-phase-noise microwave sources based on optoelectronic oscillators for high-frequency and high-performance applications.

\section{How can the field be improved?}

The integration of microwave photonic components in photonic-integrated circuits is crucial to the implementation of low-cost, advanced microwave photonics. Although microwave photonics has unique features not offered by state-of-the-art electronics, its applications are still limited because of high manufacturing costs. Recent activity in silicon photonics (photonic devices produced within a standard silicon foundry using standard silicon processing) to integrate both active and passive photonic devices in a silicon chip may help to realize integrated microwave photonics systems at significantly reduced cost and with greatly improved performance. Although the performance of silicon photonic devices is advancing at an ever-increasing pace, more effort is still needed to design and implement microwave photonic circuits on a chip for practical applications.

\section{RACHEL WON}

Rachel Won is a senior editor of Nature Photonics in Tokyo, Japan. 